\title{
Simulation of optical interstellar scintillation
}

\author{
F. Habibi ${ }^{1,2}$, M. Moniez ${ }^{1}$, R. Ansari ${ }^{1}$, and S. Rahvar ${ }^{3,4}$ \\ ${ }^{1}$ Laboratoire de l'Accélérateur Linéaire, IN2P3 - CNRS, Université de Paris-Sud, BP 34, 91898 Orsay Cedex, France \\ e-mail: moniez@lal.in2p3.fr \\ 2 School of Astronomy, Institute for Research in Fundamental Sciences (IPM), PO Box 19395-5531,Tehran, Iran \\ 3 Department of Physics, Sharif University of Technology, PO Box 11365-9161, Tehran, Iran \\ ${ }^{4}$ Perimeter Institute for Theoretical Physics, 31 Caroline Street North, Waterloo, Ontario N2L 2y5, Canada
}

Received 6 August 2012 / Accepted 6 February 2013

ABSTRACT

\begin{abstract}
Aims. Stars twinkle because their light propagates through the atmosphere. The same phenomenon is expected on a longer time scale when the light of remote stars crosses an interstellar turbulent molecular cloud, but it has never been observed at optical wavelengths. The aim of the study described in this paper is to fully simulate the scintillation process, starting from the molecular cloud description as a fractal object, ending with the simulations of fluctuating stellar light curves.

Methods. Fast Fourier transforms are first used to simulate fractal clouds. Then, the illumination pattern resulting from the crossing of background star light through these refractive clouds is calculated from a Fresnel integral that also uses fast Fourier transform techniques. Regularisation procedure and computing limitations are discussed, along with the effect of spatial and temporal coherency (source size and wavelength passband).

Results. We quantify the expected modulation index of stellar light curves as a function of the turbulence strength - characterised by the diffraction radius $R_{\text {diff }}$ - and the projected source size, introduce the timing aspects, and establish connections between the light curve observables and the refractive cloud. We extend our discussion to clouds with different structure functions from Kolmogorovtype turbulence.

Conclusions. Our study confirms that current telescopes of $\sim 4 \mathrm{~m}$ with fast-readout, wide-field detectors have the capability of discovering the first interstellar optical scintillation effects. We also show that this effect should be unambiguously distinguished from any other type of variability through the observation of desynchronised light curves, simultaneously measured by two distant telescopes.
\end{abstract}

Key words. dark matter - Galaxy: disk - Galaxy: halo - Galaxy: structure - local insterstellar matter - ISM: molecules

\section{Introduction}

This paper is a companion paper to the observational results published in Habibi et al. (2011), and it focusses on the simulation of the scintillation effects that were searched for. Cold transparent molecular clouds are one of the last possible candidates for the missing baryons of cosmic structures on different scales (Pfenniger \& Combes 1994; Pfenniger \& Revaz 2005 and McGaugh et al. 2010). Since these hypothesised clouds do not emit or absorb light, they are invisible for the terrestrial observer, so we have to investigate indirect detection techniques. Our proposal for detecting such transparent clouds is to search for the scintillation of the stars located behind the transparent medium, caused by the turbulence of the cloud (Moniez 2003 and Habibi et al. 2011). The objective of this technical paper is to describe the way we can connect observations to scintillation parameters through a realistic simulation. We used these connections in the companion paper (Habibi et al. 2011) to establish constraints both from null results (towards SMC) and from observations pointing to a possible scintillation effect (towards nebula B68). Similar studies of propagation through a stochastic medium followed by Fresnel diffraction have been made by Coles et al. (1995) and for use in radio-astronomy by Hamidouche \& Lestrade (2007).

We first introduce the notations and the formalism in Sect. 2. Then we describe the different stages of the simulation pipeline up to the production of simulated light curves in Sect. 3. We study the observables that can be extracted from the light curve of a scintillating star, and in particular, we check the expected modulation amplitude properties in Sect. 4. The discussion is extended to non-Kolmogorv turbulence cases in Sect. 5. In Sect. 6 we use the results from the simulation pipeline to optimise the observational strategy for discovering scintillating stars, and indicate some perspectives in the conclusion.

Complementary information on observations made with the ESO-NTT telescope and on the analysis based on the present simulations are to be found in our companion paper (Habibi et al. 2011).

\section{Basic definitions and formalism}

The formalism described in this section has been inspired and adapted from the radioastronomy studies (Narayan 1992). But at optical wavelength, the scintillation is primarily due to the refraction through dense clouds of $\mathrm{H}_{2}+\mathrm{He}$ instead of the interaction with the ionised interstellar medium. The origin of the stochastic phase fluctuation experienced by the electromagnetic wave when crossing the refractive medium is the phase excess induced by the stochastic fluctuation of the column density due to the turbulence (Moniez 2003):

$\phi\left(x_{1}, y_{1}\right)=\frac{(2 \pi)^{2} \alpha}{\lambda} N l\left(x_{1}, y_{1}\right)$

where $x_{1}$ and $y_{1}$ are the coordinates in the cloud's plane, perpendicular to the sightline (see Fig. 1). Here $\phi\left(x_{1}, y_{1}\right)$ is the phase delay induced to the wavefront after crossing the cloud, $N l\left(x_{1}, y_{1}\right)$ is the cloud column density of $\mathrm{H}_{2}$ molecules plus He atoms along 


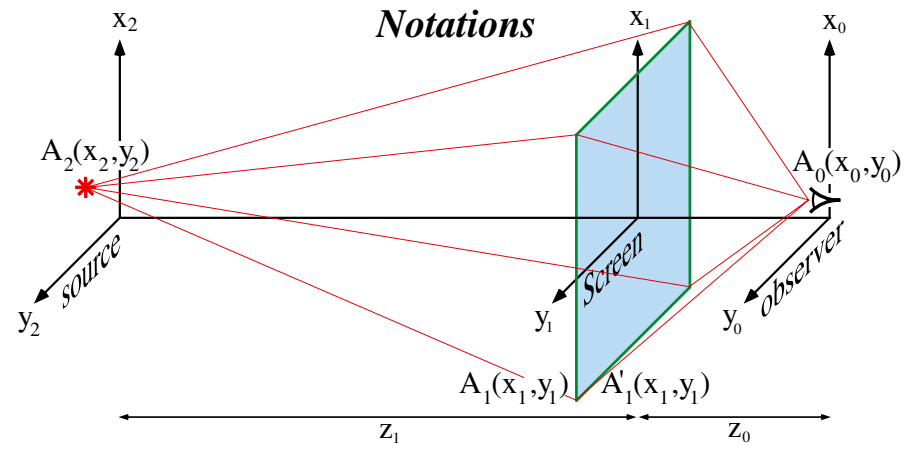

Fig. 1. Geometric configuration. The source is located in the $\left(x_{2}, y_{2}\right)$ plane, the screen contains the refractive structure, and the observer is located in the $\left(x_{0}, y_{0}\right)$ plane. $A_{1}\left(x_{1}, y_{1}\right)$ and $A_{1}^{\prime}\left(x_{1}, y_{1}\right)$ are the amplitudes before and after screen crossing.

the line of sight, $\alpha$ is the medium polarisability, and $\lambda$ the wavelength. The phase delay here scales with $\lambda^{-1}$, in contrast to the radioastronomy where it scales with $\lambda$. Since other sources of phase delay, such as the geometrical delay induced by scattering from cloud inhomogeneities, are negligible, the thin screen approximation can be used, and the cloud can be considered as a $2 \mathrm{D}$ scattering screen whose optical properties are mapped by the phase screen $\phi\left(x_{1}, y_{1}\right)$. The statistical properties of the phase screen are described by the phase structure function $D_{\phi}\left(x_{1}, y_{1}\right)$. By assuming an isotropic turbulence (Narayan 1992):

$D_{\phi}\left(x_{1}, y_{1}\right)=D_{\phi}(r)$

$=\left\langle\left[\phi\left(x_{1}+x_{1}^{\prime}, y_{1}+y_{1}^{\prime}\right)-\phi\left(x_{1}^{\prime}, y_{1}^{\prime}\right)\right]^{2}\right\rangle_{\left(x_{1}^{\prime}, y_{1}^{\prime}\right)}=\left[\frac{r}{R_{\text {diff }}}\right]^{\beta-2}$,

where the first expression is averaged over the plane positions $\left(x_{1}^{\prime}, y_{1}^{\prime}\right), r=\sqrt{x_{1}^{2}+y_{1}^{2}}, \beta$ is the turbulence exponent - equals $11 / 3$ for Kolmogorov turbulence - and the diffraction radius, $R_{\text {diff }}$, is the transverse distance on the phase screen for which the root mean square of the phase variation in one radian. The diffraction radius can be expressed in terms of the cloud parameters (Habibi et al. 2011); assuming Kolmogorov turbulence it is given by

$R_{\mathrm{diff}}(\lambda)=263 \mathrm{~km}\left[\frac{\lambda}{1 \mu \mathrm{m}}\right]^{\frac{6}{5}}\left[\frac{L_{z}}{10 \mathrm{AU}}\right]^{-\frac{3}{5}}\left[\frac{L_{\mathrm{out}}}{10 \mathrm{AU}}\right]^{\frac{2}{5}}\left[\frac{\sigma_{3 n}}{10^{9} \mathrm{~cm}^{-3}}\right]^{-\frac{6}{5}}$

where $L_{z}$ is the cloud size along the sightline, $L_{\mathrm{out}}$ is the turbulence outer scale, and $\sigma_{3 n}$ is the dispersion of the volumic number density in the medium ${ }^{1}$. Here we assume the gas to be a mixing of $\mathrm{H}_{2} / \mathrm{He}$ with $24 \% \mathrm{He}$ by mass (corresponding to the primordial abundances) and therefore $\langle\alpha\rangle=0.720 \times 10^{-30} \mathrm{~m}^{3}$. In this expression, the cloud parameters are scaled to the values given by the Pfenniger-Combes model for the clumpuscules (the tiniest cloudlets of the molecular cloud). In the NIR band, the diffraction radius of a typical clumpuscule is expected to be $R_{\text {diff }} \sim 500 \mathrm{~km}$.

The phase statistics of the screen can be equivalently described in Fourier space by the phase spectral density:

$S_{\phi}\left(q_{x}, q_{y}\right)=S_{\phi}(q)=\frac{R_{\mathrm{diff}}^{2}}{2(2 \pi)^{\beta-1} f(\beta)}\left(R_{\mathrm{diff}} q\right)^{-\beta}$,

\footnotetext{
1 A cloud column of width $L_{z}$ can include several turbulent structures with outer scale $L_{\text {out }}$. The direct relation of $L_{\text {out }}$ with the turbulence strengh explains why $R_{\text {diff }}$ increases in conjunction with this parameter. By contrast, since the column density increases with $L_{z}$, then the refraction also increases, thus decreasing $R_{\text {diff }}$.
}

where Fourier coordinates $q_{x}$ and $q_{y}$ have inverse length dimension, $q=\sqrt{q_{x}^{2}+q_{y}^{2}}$, and $f(\beta)=\frac{2^{-\beta} \beta \Gamma(-\beta / 2)}{\Gamma(\beta / 2)}$ is a constant.

After crossing the cloud, the distorted wavefront of a point source propagates toward the observer and produces an illumination pattern on the observer's plane given by

$I_{0}\left(x_{0}, y_{0}\right)=\frac{L_{\mathrm{s}}}{z_{1}^{2}} h\left(x_{0}, y_{0}\right)$,

where $I_{0}\left(x_{0}, y_{0}\right)$ is the light intensity on the observer's plane, $L_{\mathrm{s}}$ is the source luminosity, $z_{1}$ is the source-screen distance (see Fig. 1), and $h\left(x_{0}, y_{0}\right)$ is given by the Fresnel-Huygens diffraction integral after considering the Fresnel and the stationary phase approximations (Born \& Wolf 2002; Moniez 2003):

$h\left(x_{0}, y_{0}\right)=\left|\frac{1}{2 \pi R_{\mathrm{F}}^{2}} \iint_{-\infty}^{+\infty} \mathrm{e}^{\mathrm{i} \phi\left(x_{1}, y_{1}\right)} \mathrm{e}^{\mathrm{i} \frac{\left(x_{0}-x_{1}\right)^{2}+\left(y_{0}-y_{1}\right)^{2}}{2 R_{\mathrm{F}}^{2}}} \mathrm{~d} x_{1} \mathrm{~d} y_{1}\right|^{2}$,

where $R_{\mathrm{F}}=\sqrt{\lambda z_{0} / 2 \pi}$ is the Fresnel radius ${ }^{2}$ and $z_{0}$ is the screenobserver distance. The Fresnel radius can be expressed as

$R_{\mathrm{F}}=2214 \mathrm{~km}\left[\frac{\lambda}{1 \mu \mathrm{m}}\right]^{\frac{1}{2}}\left[\frac{z_{0}}{1 \mathrm{kpc}}\right]^{\frac{1}{2}}$

At the typical distance of a halo object ( $\sim 10 \mathrm{kpc}), R_{\mathrm{F}} \sim 7000 \mathrm{~km}$ for NIR wavelengths. Because of the motion of the cloud with respect to the Earth-source line-of-sight, the illumination pattern sweeps the observer plane, so that a terrestrial observer receives fluctuating intensity light from the point source. This effect, the scintillation, has two different scattering regimes (Uscinski 1977; Tatarskii \& Zavorotnyi 1980), a weak regime $\left(R_{\text {diff }}>R_{\mathrm{F}}\right)$ and a strong regime $\left(R_{\text {diff }}<R_{\mathrm{F}}\right)$. In the present studies, we concentrate on the strong regime, which clearly is easier to detect, but some realistic configurations may involve the intermediate regime studied by Goodman \& Narayan (2006). For the strong regime, there are two different modes of flux variations (Narayan 1992; see also Rickett 1986; Rumsey 1975; Sieber 1982). The first one is the diffractive mode with length scale corresponding to the screen's scale of phase variations $R_{\text {diff }}$ given by Eq. (3). The resulting "speckles", with typical size of the order of $R_{\text {diff }}$, are shown in Fig. 4 . The corresponding time scale of the light fluctuations is $t_{\mathrm{diff}}=R_{\mathrm{diff}} / V_{\mathrm{T}}$ :

$t_{\text {diff }}(\lambda)=2.6 \mathrm{~s}$

$\times\left[\frac{\lambda}{1 \mu \mathrm{m}}\right]^{\frac{6}{5}}\left[\frac{L_{z}}{10 \mathrm{AU}}\right]^{-\frac{3}{5}}\left[\frac{L_{\mathrm{out}}}{10 \mathrm{AU}}\right]^{\frac{2}{5}}\left[\frac{\sigma_{3 n}}{10^{9} \mathrm{~cm}^{-3}}\right]^{-\frac{6}{5}}\left[\frac{V_{\mathrm{T}}}{100 \mathrm{~km} \mathrm{~s}^{-1}}\right]^{-1}$,

where $V_{\mathrm{T}}$ is the sightline relative transverse motion. Therefore fast flux variations are expected with a typical time scale of $t_{\text {diff }} \sim$ few s. The second variation mode is the refractive mode associated to the longer length scale called refraction radius:

$R_{\text {ref }}(\lambda)=\frac{\lambda z_{0}}{R_{\text {diff }}(\lambda)} \sim 30860 \mathrm{~km}\left[\frac{\lambda}{1 \mu \mathrm{m}}\right]\left[\frac{z_{0}}{1 \mathrm{kpc}}\right]\left[\frac{R_{\mathrm{diff}}(\lambda)}{1000 \mathrm{~km}}\right]^{-1}$.

This natural length scale corresponds to the size, in the observer's plane, of the diffraction spot from a patch of $R_{\text {diff }}(\lambda)$ in the screen's plane. This is also the size of the region in the screen where most of the scattered light seen at a given observer's position originates. Our convention for $R_{\text {ref }}$ differs from Narayan (1992) by a factor $2 \pi$ since it emerges naturally from 2 This definition assumes that $z_{0} \ll z_{1}$. In the general case, $z_{0}^{-1}$ should
be replaced by $z_{0}^{-1}+z_{1}^{-1}$. 
the Fourier transform we use for calculating the illumination pattern (see formula (14)), and it also matches the long distancescale flux variations visible in Fig. 4. The corresponding time scale is given by $t_{\text {ref }}=R_{\text {ref }} / V_{\mathrm{T}}$ :

$t_{\mathrm{ref}}(\lambda) \simeq 5.2 \mathrm{~min}\left[\frac{\lambda}{1 \mu \mathrm{m}}\right]\left[\frac{z_{0}}{1 \mathrm{kpc}}\right]\left[\frac{R_{\mathrm{diff}}(\lambda)}{1000 \mathrm{~km}}\right]^{-1}\left[\frac{V_{\mathrm{T}}}{100 \mathrm{~km} \mathrm{~s}^{-1}}\right]^{-1}$.

\section{Simulation description}

In this section, we describe the simulation pipeline with some numerical tricks, from the generation of the phase screen induced by turbulent gas, up to the light versus time curves expected from realistic stars seen through this gas. The steps in this pipeline are listed below:

- The simulation of the refractive medium: in Sect. 3.1 we describe the generation of a phase screen and examine the impact of the limitations caused by the sampling and by the finite size of the screen by comparing the initial (theoretical) and reconstructed diffraction radii.

- The computation of the illumination pattern: in Sect. 3.2, we first describe the calculation of the illumination pattern produced on Earth by a point monochromatic source as seen through the refractive medium. We explain the technique for avoiding numerical (diffraction) artefacts caused by the borders of the simulated screen, and discuss the criterion on the maximum pixel size to avoid aliasing effects. The pattern computation is then generalised to extended polychromatic sources.

- The light curve simulation: we describe in Sect. 3.3 the simulation of the light fluctuations with time at a given position induced by the motion of the refractive medium with respect to the line of sight.

\subsection{Simulation of the phase screen}

Numerical realisations of the 2D phase screen - made of $N_{x} \times N_{y}$ squared pixels of size $\Delta_{1}$ - are randomly generated from the phase spectral density $S_{\phi}\left(q_{x}, q_{y}\right)$, determined by the choice of $R_{\text {diff }}$ in relation (4). Such a phase screen - with the desired statistical properties - is obtained from the random realisation of a Fourier transform $F_{\phi}\left(q_{x}, q_{y}\right)$ in a way that makes the ensemble of such realisations satisfy the relation

$$
\left\langle\left|F_{\phi}\left(q_{x}, q_{y}\right)\right|^{2}\right\rangle_{\text {realisations }}=L_{x} L_{y} S_{\phi}\left(q=\sqrt{q_{x}^{2}+q_{y}^{2}}\right),
$$

where $L_{x}$ and $L_{y}$ are the screen physical size, and the average is the ensemble averaging over the different realisations. For each $\left(q_{x}^{i}, q_{y}^{j}\right)$ vector associated to a pixel $(i, j)$, we generate a random complex number $F_{\phi}\left(q_{x}^{i}, q_{y}^{j}\right)=\left(f_{\mathrm{Re}}^{i, j}+\mathrm{i} f_{\mathrm{Im}}^{i, j}\right) / \sqrt{2}$ where each component $f_{\mathrm{Re}}^{i, j}$ and $f_{\mathrm{Im}}^{i, j}$ spans the Gaussian distribution ${ }^{3}$ of zero mean, and $L_{x} L_{y} S_{\phi}\left(q=\sqrt{q_{x}^{i 2}+q_{y}^{j 2}}\right)$ width. In this way, relation 11 is automatically satisfied when averaging on a large number of such realisations, since the average of $\left|F_{\phi}\left(q_{x}, q_{y}\right)\right|^{2}$ for the ensemble of $\left(q_{x}, q_{y}\right)$ vectors with the same module $q$ equals $L_{x} L_{y} S_{\phi}(q)$ by construction. The phase screen $\phi\left(x_{1}, y_{1}\right)$ is finally obtained by numerically computing the inverse Fourier transform of this phase spectrum $F_{\phi}\left(q_{x}, q_{y}\right)$. Figure 2 shows a phase screen generated by assuming Kolmogorov turbulence $(\beta=11 / 3)$.

\footnotetext{
3 The turbulence is considered as a Gaussian field.
}

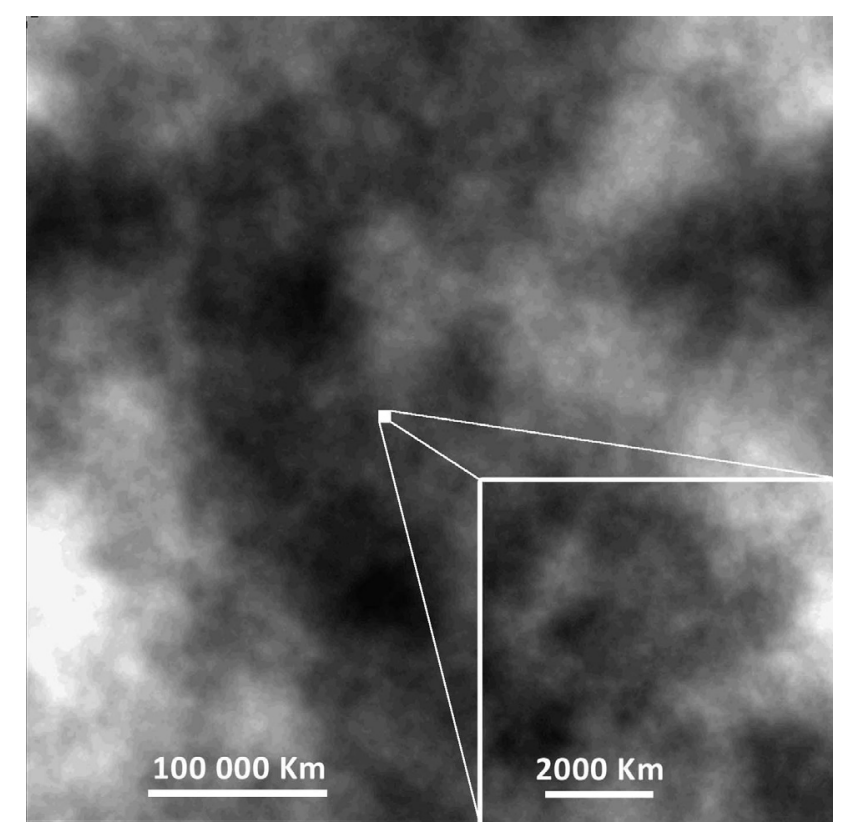

Fig. 2. The phase-delay variations near the average for a simulated refractive screen with $N_{x} \times N_{y}=20000 \times 20000$ pixels, $\Delta_{1}=22.6 \mathrm{~km}$, and $R_{\text {diff }}=100 \mathrm{~km}$. The grey scale ranges between $\pm 50 \times 2 \pi \mathrm{rad}$ (clear regions correspond to an excess of phase with respect to the average). The zoom (inset in the lower-right corner) illustrates the self-similarity of the simulated screen (grey scale amplitude of $5 \times 2 \pi \mathrm{rad}$ ).

\subsubsection{Preliminary checks, limitations}

To check the accuracy of the numerically generated phase screen (Fig. 2), we recomputed the phase structure function $D_{\phi}^{\text {rec }}(r)$ (and consequently $R_{\text {diff }}^{\text {rec }}$ ) from the generated phase Fourier transform $F_{\phi}\left(q_{x}, q_{y}\right)$, and we compared it with the theoretical phase structure function (Eq. (2)). First, the spectral density is recomputed from the generated $F_{\phi}\left(q_{x}, q_{y}\right)$ using relation

$S_{\phi}^{\mathrm{rec}}(q)=\frac{\left\langle\left|F_{\phi}\left(q_{x}, q_{y}\right)\right|^{2}\right\rangle_{q=\sqrt{q_{x}^{2}+q_{y}^{2}}}}{L_{x} L_{y}}$

where the average is performed on the $\left(q_{x}, q_{y}\right)$ coordinates $^{4}$ spanning the circle of radius $q=\sqrt{q_{x}^{2}+q_{y}^{2}}$. The corresponding phase auto-correlation function is then given by Fourier transform:

$$
\begin{aligned}
\xi^{\mathrm{rec}}(\mathbf{r}) & =\iint S_{\phi}^{\mathrm{rec}}(\mathbf{q}) \mathrm{e}^{2 \pi \mathrm{i} \mathbf{q} \cdot \mathbf{r}} \mathrm{d} \mathbf{q}, \\
\xi^{\mathrm{rec}}(r) & =\int_{q_{\min }}^{q_{\max }} \int_{0}^{2 \pi} q S_{\phi}^{\mathrm{rec}}(q) \mathrm{e}^{2 \pi \mathrm{i} q r \cos \theta} \mathrm{d} \theta \mathrm{d} q \\
& =\int_{q_{\min }}^{q_{\max }} 2 \pi q S_{\phi}^{\mathrm{rec}}(q) J_{0}(2 \pi q r) \mathrm{d} q,
\end{aligned}
$$

where $J_{0}$ is the Bessel function. The recomputed structure function is then given by $D_{\phi}^{\mathrm{rec}}(r)=2\left(\xi^{\mathrm{rec}}(0)-\xi^{\mathrm{rec}}(r)\right)$, and the value of $R_{\text {diff }}^{\text {rec }}$ is deduced from its definition $D_{\phi}^{\text {rec }}\left(R_{\text {diff }}^{\text {rec }}\right)=1$.

In Fig. 3, we show the theoretical phase structure function of a turbulent medium with $R_{\text {diff }}=500 \mathrm{~km}-$ for which

4 Here again, we infer the ergodicity property that allows us to replace the ensemble averaging with an average on directions from only one realisation. 


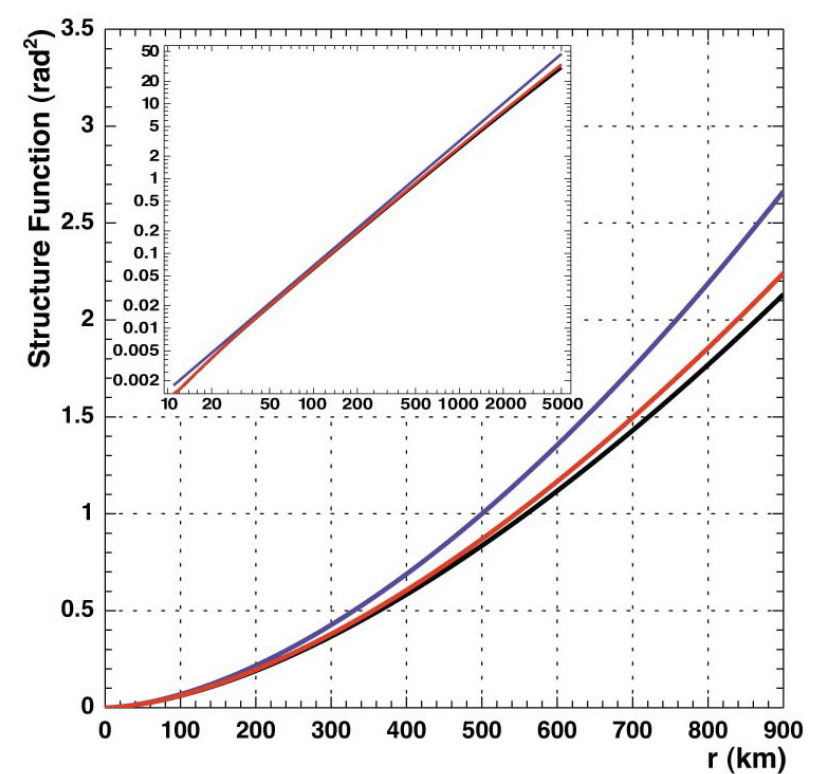

Fig. 3. Phase structure functions $D_{\phi}(r)$ for a phase screen with $R_{\text {diff }}=$ $500 \mathrm{~km}$. Blue line is the initial (theoretical) structure function. Red line is reconstructed from one of the realisations of the phase screen through simulation. The black curve is obtained from the numerical integration of the initial phase spectral density sampled as in the simulation.

$D_{\phi}(r=500 \mathrm{~km})=1$ by definition - , and the recomputed (effective) structure function from one of the realisations of the screen. From this recomputed function, we find $R_{\mathrm{diff}}^{\mathrm{rec}} \approx 540 \mathrm{~km}$ since $D_{\phi}^{\mathrm{rec}}(r \approx 540 \mathrm{~km})=1$. To find the origin of the difference with the input value $R_{\text {diff }}=500 \mathrm{~km}$, we replaced $S_{\phi}^{\mathrm{rec}}(q)$ in Eq. (13) by the theoretical spectrum $S_{\phi}(q)$ sampled as in the simulation (number of pixels $N_{x} \times N_{y} \sim 14000 \times 14000$ with pixel size $\Delta_{1}=28.85 \mathrm{~km}$ ). Then we computed the integral (13) numerically with the same integration limits $\left(q_{\min }, q_{\max }\right)^{5}$ as the simulation. The integration result differs only by a few percent from the function recomputed from the simulated screen. We showed that the black curve approaches the blue curve when $q_{\min } \rightarrow 0$ and $q_{\max } \rightarrow \infty$. This means that the sampling is mainly responsible for the difference between $D_{\phi}$ and $D_{\phi}^{\text {rec }}$. Since our simulation is limited by the number of pixels, we lose the contributions of the large and small scales in the recomputed $R_{\text {diff }}$. The only way to push back this limitation is to generate larger screens (larger $N_{x}$ and $N_{y}$ ) with higher resolutions (smaller $\Delta_{1}$ ) to cover wider interval of spatial frequencies which in return needs higher computational capacities (see also Sect. 3.4).

\subsection{Illumination pattern}

To obtain the illumination pattern on the observer plane, we numerically compute the integral (6) which can be written as a Fourier transform:

$h\left(x_{0}, y_{0}\right)=\frac{1}{2 \pi R_{\mathrm{F}}^{2}}\left|F T\left[G\left(x_{1}, y_{1}\right)\right]\right|^{2}\left(f_{x}=\frac{x_{0}}{2 \pi R_{\mathrm{F}}^{2}}, f_{y}=\frac{y_{0}}{2 \pi R_{\mathrm{F}}^{2}}\right)$,

where

$G\left(x_{1}, y_{1}\right)=\exp \left[\mathrm{i}\left(\phi\left(x_{1}, y_{1}\right)+\frac{x_{1}^{2}+y_{1}^{2}}{2 R_{\mathrm{F}}^{2}}\right)\right]$.

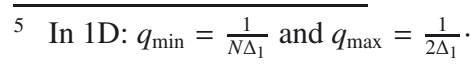

A93, page 4 of 10
Here, $\left(x_{1}, y_{1}\right)$ and $\left(x_{0}, y_{0}\right)$ are the screen and observer coordinates, respectively. Coordinates $\left(f_{x}, f_{y}\right)$ are the conjugated variables in Fourier space. Before computing expression (14), a regularisation procedure for $G\left(x_{1}, y_{1}\right)$ has been defined to avoid computational artefacts.

\subsubsection{Screen regularisation}

Since integral (14) is computed numerically, the coordinates $\left(x_{1}, y_{1}\right)$ have discrete (integer) values describing pixel position centres on the screen, to allow simple combinations of illumination patterns with different pixel sizes (corresponding to different wavelengths). That the integration domain is limited is physically equivalent to computing the Fresnel integral within a diaphragm with the size of the screen. In this case, we face a parasitic effect: the light diffraction from the sharp edges of the diaphragm. This causes rapid intensity variations at the borders of the observer plane. To attenuate this effect and remove the resulting diffraction fringes, we multiply the screen intensity transmission by a 2D smoothing function. We define the following $1 \mathrm{D}$ smoothing function $S F(x)$ (see Fig. 6):

$S F(x)= \begin{cases}\frac{1}{2}\left[1+\sin \left(\frac{\pi x}{L_{\mathrm{m}}}-\frac{\pi}{2}\right)\right] & 0 \leq x \leq L_{\mathrm{m}}, \\ 1 & L_{\mathrm{m}}<x<L-L_{\mathrm{m}}, \\ \frac{1}{2}\left[1+\sin \left(\frac{\pi\left(x-L+L_{\mathrm{m}}\right)}{L_{\mathrm{m}}}+\frac{\pi}{2}\right)\right] & L-L_{\mathrm{m}} \leq x \leq L, \\ 0 & \text { otherwise, }\end{cases}$

where $L_{\mathrm{m}}=10 R_{\mathrm{F}}$ is the margin length from the borders of the screen with size $L$. We multiply the function $G\left(x_{1}, y_{1}\right)$ by $S F\left(x_{1}\right) \times S F\left(y_{1}\right)$ in expression (14). Since the Fresnel integral is dominated by the contribution of the integrand within a disk that is within a few Fresnel radii, it is sufficient to smooth the discontinuity of $G\left(x_{1}, y_{1}\right)$ within a distance of a few Fresnel radii (here $L_{\mathrm{m}}=10 R_{\mathrm{F}}$ ). We tested the efficiency of this regularisation procedure by checking that the simulated illumination pattern from a point-source projected through a uniform phase screen was - as expected - also uniform beyond our precision requirements $(<1 \%)$ (Habibi 2011). To define a reliable fiducial domain in the observer plane excluding the regions that are only partially illuminated owing to the diaphragm, we also delimited an $R_{\text {ref }} / 2$ margin from the borders of the illumination pattern, corresponding to the typical radius (half-size) of the large-scale luminous spots (see also Coles et al. 1995). Figure 4 shows the pattern produced by a point source through a turbulent medium with $R_{\text {diff }}=100 \mathrm{~km}$ located at $z_{1}=160 \mathrm{pc}$ from the Earth at wavelength $\lambda=2.162 \mu \mathrm{m}$. Since the corresponding Fresnel radius $R_{\mathrm{F}}=1300 \mathrm{~km}$ is larger than the diffraction radius, the regime is the strong scintillation regime. The hot speckles (of typical size $R_{\text {diff }} \sim 100 \mathrm{~km}$ ) can be distinguished from the larger dark/luminous structures that have a typical size of $R_{\text {ref }} \sim 2 \pi R_{\mathrm{F}}^{2} / R_{\text {diff }} \simeq 100000 \mathrm{~km}$.

\subsubsection{Effect of sampling}

Here, we discuss some limitations caused by the pixellisation. The screen should be sampled often enough to avoid the aliasing effects. Aliasing happens when $G\left(x_{1}, y_{1}\right)$ contains frequencies that are higher than the Nyquist frequency $f_{\mathrm{Nyq}}=1 /\left(2 \Delta_{1}\right)$, where $\Delta_{1}$ is the pixel size. In relation (15), $G$ contains two length scales, the diffraction and the Fresnel radii $\left(R_{\text {diff }}\right.$ and $\left.R_{\mathrm{F}}\right) . R_{\text {diff }}$ is the 


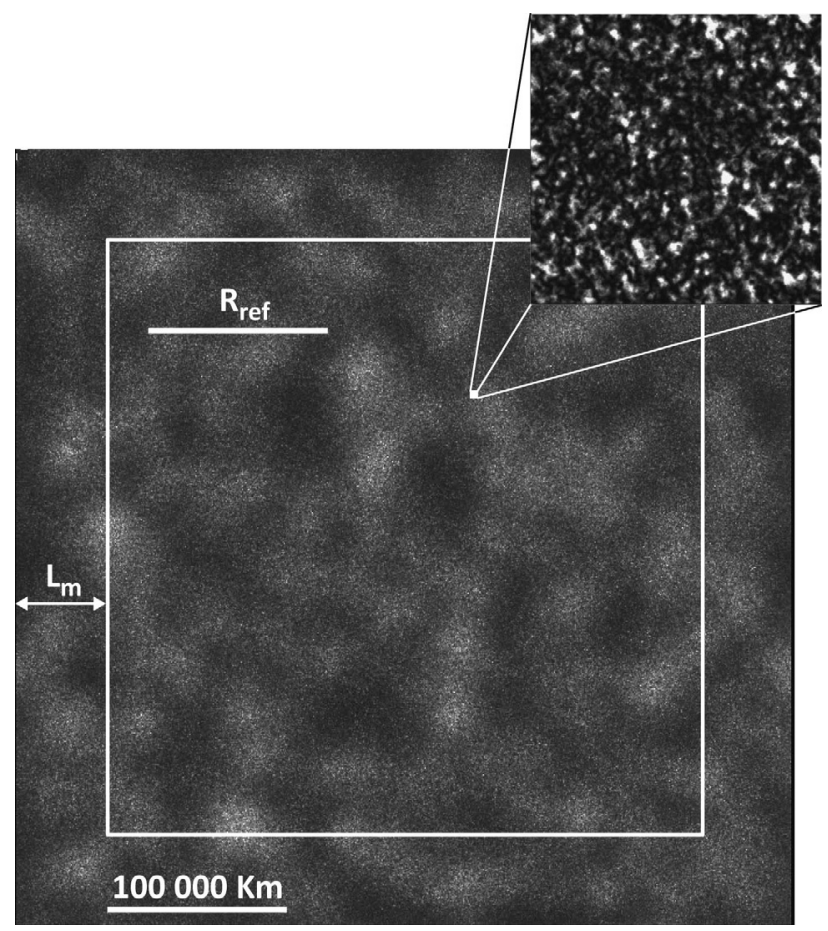

Fig. 4. Typical illumination pattern from a point-like source. Here $R_{\text {diff }}=100 \mathrm{~km}$, the screen is at $z_{0}=160 \mathrm{pc}, \lambda=2.16 \mu \mathrm{m}$, then $R_{\mathrm{F}}=1300 \mathrm{~km}$ and $R_{\text {ref }}=106000 \mathrm{~km}$. The typical length scale of the small-scale speckles is $R_{\text {diff }}$, and the scale of the larger structures is $R_{\text {ref }}$. The white square shows our fiducial zone with a margin of $L_{\mathrm{m}}=R_{\text {ref }} / 2$ from the borders. Grey scale range from 0 to 4 times the mean intensity. The image has $20000 \times 20000$ pixels, each with a $22.6 \mathrm{~km}$ side.

characteristic length of the phase screen variations $\phi\left(x_{1}, y_{1}\right)$; it is at least necessary that $\Delta_{1}<R_{\text {diff }} / 2$ in order to sample phase variations up to $1 / R_{\text {diff }}$ spatial frequency. $R_{\mathrm{F}}$ appears in the quadratic term $\exp \left[\mathrm{i} \frac{x_{1}^{2}+y_{1}^{2}}{2 R_{\mathrm{F}}^{2}}\right]$; the oscillation of this term accelerates as $x_{1}$ and $y_{1}$ increase, and aliasing occurs as soon as the distance between two consecutive peaks is smaller than $2 \Delta_{1}$. In one dimension we therefore expect aliasing if

$$
\begin{aligned}
& \frac{\left(x_{1}+2 \Delta_{1}\right)^{2}-x_{1}^{2}}{2 R_{\mathrm{F}}^{2}}>2 \pi, \\
& \text { i.e. } \frac{x_{1}}{\Delta_{1}}>\pi\left[\frac{R_{\mathrm{F}}}{\Delta_{1}}\right]^{2}-1,
\end{aligned}
$$

where $x_{1} / \Delta_{1}$ is the distance to the optical axis, expressed in pixels. The condition $\Delta_{1}=R_{\mathrm{F}} / 2$ would be obviously insufficient here to avoid aliasing, since beyond $\sim 11$ pixels only from the optical axis $\left(x_{1} / \Delta_{1}>11\right.$ pixel $)$, the quadratic term would be undersampled. In practice, for the configurations considered in this paper, the Fresnel radius is $>1000 \mathrm{~km}$. When $\Delta_{1}=22 \mathrm{~km}$ in the simulation, the aliasing starts at $x_{1} / \Delta_{1}>6490$ pixels from the centre of the image, corresponding to more than $140 R_{\mathrm{F}}$, which is large enough to cover the sensitive domain related to the stationary phase approximation.

\subsubsection{Extended source (spatial coherency)}

The illumination pattern of a scintillating extended source is given by the convolution product of the illumination pattern

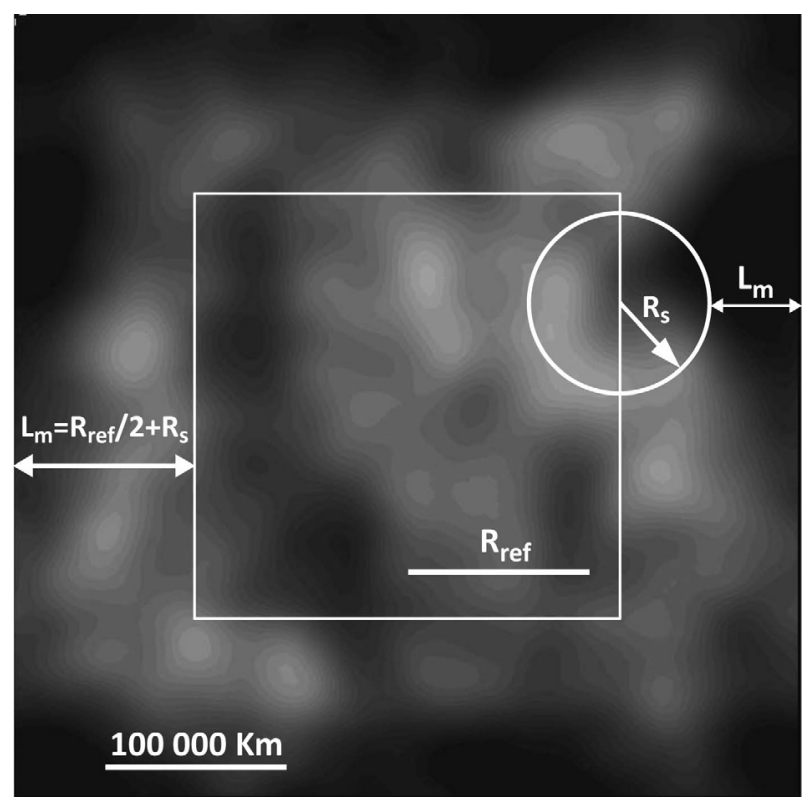

Fig. 5. Typical illumination pattern from an extended source produced through the same screen as in Fig. 4, with source radius $r_{\mathrm{s}}=0.5 R_{\odot}$, located at $z_{0}+z_{1}=1 \mathrm{kpc}+160 \mathrm{pc}\left(R_{\mathrm{s}} \simeq 53000 \mathrm{~km}\right)$. The small-scale speckles are smeared, and only the larger scale fluctuations survive. The white square shows the restricted fiducial zone with margin of $L_{\mathrm{m}}=$ $R_{\text {ref }} / 2+R_{\mathrm{S}}$ from the borders. Grey scale ranges $\pm 20 \%$ around the mean intensity.

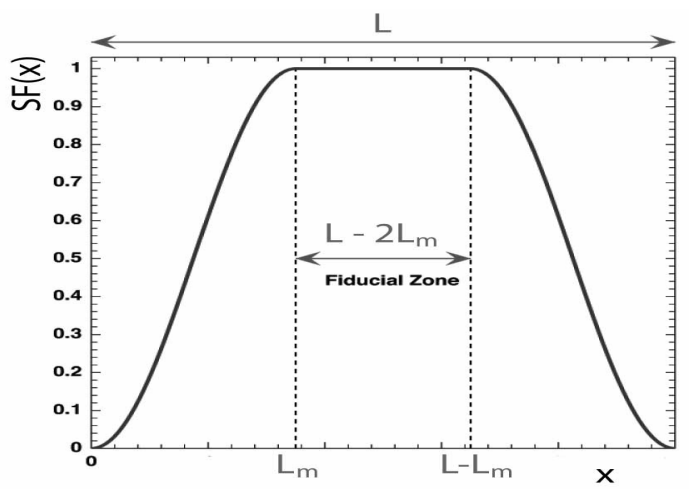

Fig. 6. The smoothing function $S F(x) . L$ is the screen size, $L_{\mathrm{m}}$ is the margin from the screen borders.

of the point-like source with the projected source limb profile (Moniez 2003; \& Habibi 2011),

$I_{\mathrm{ext}}=\frac{L_{\mathrm{s}}}{z_{0}^{2}} P_{r} * h$

where $L_{\mathrm{s}}$ is the source luminosity, and the normalised limb profile is described as a uniform disk:

$P_{r}\left(x_{0}, y_{0}\right)= \begin{cases}1 / \pi R_{\mathrm{s}}^{2} & \sqrt{x_{0}^{2}+y_{0}^{2}} \leq R_{\mathrm{s}} \\ 0 & \text { otherwise }\end{cases}$

where $R_{\mathrm{s}}=\frac{z_{0}}{z_{1}} r_{\mathrm{s}}$ is the projected source radius on the observer's plane, and $r_{\mathrm{s}}$ is the source radius. Figure 5 shows the convolution of the pattern of Fig. 4 with the projected profile of a star with radius $r_{\mathrm{s}}=0.5 R_{\odot}$, located at $z_{0}(160 \mathrm{pc})+z_{1}(1 \mathrm{kpc})=1.16 \mathrm{kpc}$ $\left(R_{\mathrm{S}}=53000 \mathrm{~km}\right)$. High-frequency fluctuations due to diffractive speckle disappear, and the pattern loses contrast, but the variations on $R_{\text {ref }}$ scale remain visible. As the convolution involves a 


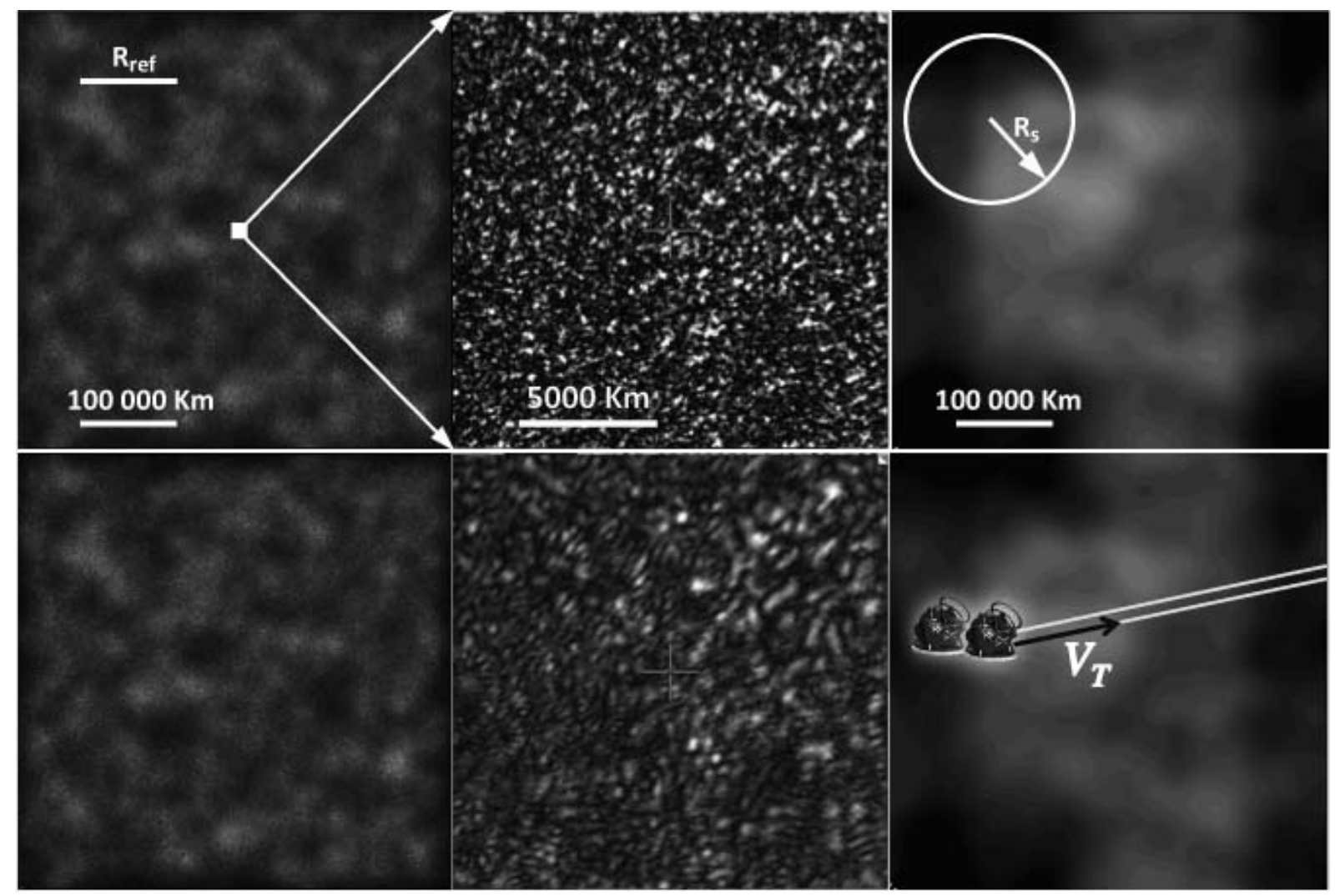

Fig. 7. Simulated illumination maps $(20000 \times 20000$ pixel of $22.6 \mathrm{~km}$ side $)$ produced on Earth by a source located at $z_{0}+z_{1}=1.18 \mathrm{kpc}$ through a refracting cloud assumed to be at $z_{0}=160 \mathrm{pc}$ with a turbulence parameter $R_{\text {diff }}(2.16 \mu \mathrm{m})=100 \mathrm{~km}$. Here $R_{\text {ref }}(2.16 \mu \mathrm{m}) \simeq 100000 \mathrm{~km}$. Top-left and middle: illumination produced at $\lambda=2.16 \mu \mathrm{m}$ from a point-source with a zoomed detail; the contrast is $100 \%$. The grey scale ranges from 0 to 4 times the mean intensity. Top-right: the same from a K0V star $\left(r_{\mathrm{s}}=0.85 R_{\odot}, M_{V}=5.9\right.$, at $\left.1.18 \mathrm{kpc} V=16.3\right)$. The circle shows the projection of the stellar disk $\left(R_{\mathrm{S}}=r_{\mathrm{s}} \times z_{0} / z_{1}\right)$. Here the modulation index is only $3.3 \%$, and the grey scale ranges from $\pm 20 \%$ around the mean intensity. The bottom maps are the illuminations in $K_{\mathrm{s}}$ wide band $\left(\lambda_{\text {central }}=2.162 \mu \mathrm{m}, \Delta \lambda=0.275 \mu \mathrm{m}\right)$, using the same grey scales as above. The modulation index is 55\% for the point-source (left and centre) and 3.3\% for the extended source (right). The two parallel straight lines show the sections sampled by two observers located about $10000 \mathrm{~km}$ apart, when the screen moves with the transverse velocity $V_{\mathrm{T}}$.

disk of radius $R_{\mathrm{s}}$, we can perform the calculation only at a distance greater than $R_{\mathrm{S}}$ from the borders. We therefore define a new fiducial zone by excluding a margin of $R_{\text {ref }} / 2+R_{\mathrm{s}}$ from the initial borders. Any statistical analysis will be made within this zone to be safe from any border perturbation.

\subsubsection{Polychromatic source (time coherency)}

The illumination patterns shown in Figs. 4 and 5 are computed for a monochromatic source (fixed $\lambda$ ), but observations are done through filters with finite-width passbands. To take the contributions of different wavelengths to the pattern into account, we superimpose the illumination patterns obtained with the same refractive structure (the same column density fluctuations) at different wavelengths ${ }^{6}$. We have considered the passband of the SOFI camera in $K_{\mathrm{s}}$ band and approximated it as a rectangular function over the transmitted wavelengths with a central value $2.162 \mu \mathrm{m}$ and width $0.275 \mu \mathrm{m}$. Twenty-one illumination patterns were computed for 21 regularly spaced wavelengths within the $[2.08,2.28] \mu \mathrm{m}$ interval, and were co-added to simulate the illumination pattern through the $K_{\mathrm{s}}$ passband. We checked that the spacing between successive wavelengths was small enough to produce a co-added image with a realistic residual modulation index, by studying this index as a function of the number

\footnotetext{
6 For a given physical screen characterised by the column density $N l\left(x_{1}, y_{1}\right), R_{\text {diff }}$ varies with $\lambda^{6 / 5}$, as shown in Eq. (3).
}

of monochromatic components equally spaced within the full bandwidth. We found that an asymptotic value is reached with a co-added image made up of only about ten components.

Figure 7 (left and centre) shows a comparison between monochromatic (up) and $K_{\mathrm{s}}$ passband (down) illumination patterns of a point-like source. The speckle pattern is attenuated when the light is not monochromatic (or equivalently when time coherency is limited). This is caused by the small decorrelation bandwidth $\delta \lambda_{\mathrm{dec}}$ of the strong diffractive scintillation regime, which is given by

$\delta \lambda_{\mathrm{dec}} / \lambda \sim\left(R_{\mathrm{diff}}(\lambda) / R_{\mathrm{F}}\right)^{2}$

(Narayan 1992; Gwinn et al. 1998). This chromatic effect results from the high sensitivity of the constructive interference condition with the wavelength ${ }^{7}$. In the case of Fig. 7, the decorrelation bandwidth is $\delta \lambda_{\mathrm{dec}} / \lambda \sim 1 \%$. Since the $K_{\mathrm{s}}$ passband is $\delta \lambda / \lambda \sim 0.1 \sim 10 \times \delta \lambda_{\mathrm{dec}} / \lambda$, a first-order estimation of the modulation index for a $K_{\mathrm{s}}$ passband pattern is the value obtained when adding ten decorrelated patterns of speckle i.e. one multiplied

\footnotetext{
7 Nevertheless, one should consider that the gaseous screen is a nondispersive medium for optical wavelengths (dielectric medium with an index independent of $\lambda$ ), unlike the radioastronomy case (plasma), which may result in weaker chromaticity sensitivity. This specificity deserves more detailed studies, which are beyond the scope of this paper, considering the minor impact of the bandwidth compared to the smearing produced by the source size.
} 
by $\sim \sqrt{1 / 10}$. The modulation index found with our simulation $(\sim 55 \%)$ has the correct order of magnitude.

By contrast, structures of size of $R_{\text {ref }}$ are much less sensitive to the variations in $\lambda$, according to the combination of (3) and (9):

$\frac{\Delta R_{\mathrm{ref}}(\lambda)}{R_{\mathrm{ref}}(\lambda)}=\frac{1}{5} \times \frac{\Delta \lambda}{\lambda}=\frac{1}{5} \times 10 \% \sim 2 \%$.

As a consequence of the large-scale smearing of the point-source pattern when considering an extended source, there is no significant difference between monochromatic and polychromatic patterns for such an extended source, as shown in Fig. 7 (right). Therefore, in the following we ignore the impact of the $R_{\mathrm{diff}}$ structures.

\subsection{Simulation of light curves}

What we observe with a single telescope is not the 2D illumination pattern but a light curve. Because of the relative motions, the telescope sweeps a 1D section of the pattern, at a constant velocity as long as parallax can be neglected. We therefore simulated light versus time curves by sampling the $2 \mathrm{D}$ pattern pixels along straight lines, with the relative speed of the telescope.

\subsection{Computing limitations}

We adopted the same number of pixels $N \times N$ and the same pixel scale $\Delta_{1}$ to numerically describe both the screen's and the observer's planes; indeed, the light emerging from the screen is essentially contained in its shadow, and this choice optimises the screen filling. Since the two planes are conjugated, the following relation arises:

$N \Delta_{1}^{2}=2 \pi R_{\mathrm{F}}^{2}$

Because of this relation, $N$ has to be as large as possible to minimize $\Delta_{1}$ and also to get a wide useful area. This area is indeed restricted by the definition of the fiducial domain, which can be heavily reduced when simulating the illumination from an extended source. Also a large fiducial domain is essential when simulating long-duration light curves. As a consequence, memory limitations affect the maximum size of the screen and illumination $2 \mathrm{D}$ patterns. In the present paper, we were limited to patterns of $20000 \times 20000$ pixels.

\section{Observables}

The observable parameters of the scintillation process are the modulation index and the modulation's characteristic time scales. The main observable we used in our data analysis (Habibi et al. 2011) is the modulation index. It is defined as the flux dispersion $\sigma_{I}$ divided by the mean flux $\bar{I}: m=\sigma_{I} / \bar{I}$. We first compare the effective modulation index of simulated screens with the theoretical expectations, then examine the precision we can reach on $m$ from a light curve, and give a numerical example. We also show how we can connect the observed modulation index to the geometrical parameters $\left(R_{\text {diff }}(\lambda), \lambda, R_{\mathrm{s}}\right.$, etc. $)$ through simulation.

\subsection{Modulation Index}

For a point-like source in the strong scintillation regime the modulation index $m \approx 1$. For an extended source (radius $r_{\mathrm{s}}$, projected

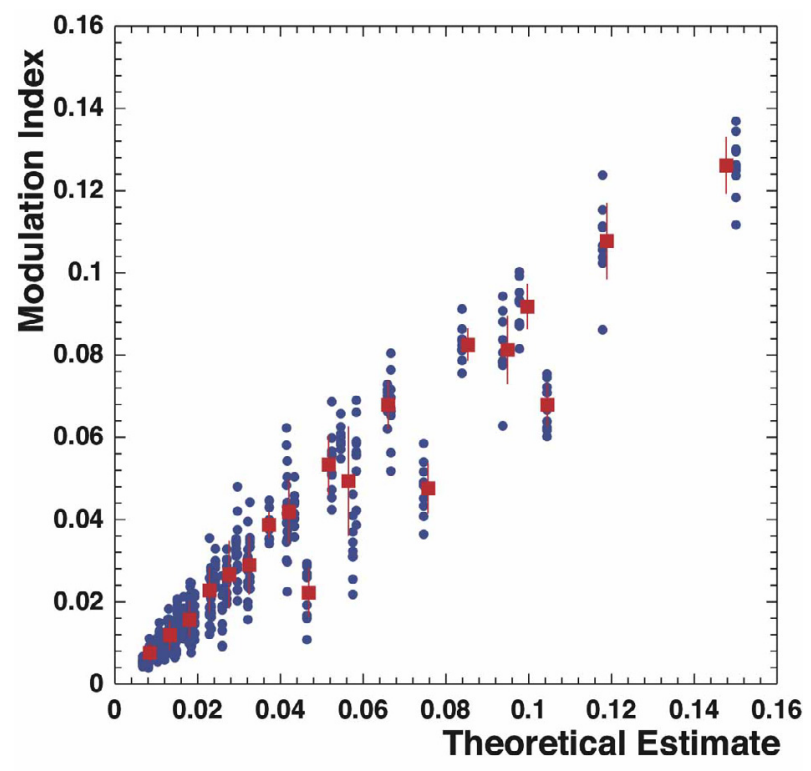

Fig. 8. The effective intensity modulation index $m=\sigma_{I} / \bar{I}$ for simulated scintillating stellar illumination patterns as a function of the theoretical modulation index. The blue dots show the effective modulation index values for different realisations of the phase screens. The red squares represent the mean value of the effective modulation indices with the same theoretical value. This plot shows the expected agreement for $m<$ 0.15 , a domain where the simulated screens are large enough not to suffer from statistical biases (see text).

radius $\left.R_{\mathrm{S}}=r_{\mathrm{s}} \cdot z_{0} / z_{1}\right)$ in the same regime, we always have $m<1$, and Narayan (1992) showed that when the small-scale (diffractive) speckle is completely smeared,

$m \approx\left[\frac{R_{\mathrm{diff}}}{R_{\mathrm{F}}}\right]^{1 / 3}\left[\frac{\theta_{\mathrm{ref}}}{2 \pi \theta_{\mathrm{s}}}\right]^{7 / 6}$

in the case of the Kolmogorov turbulence. Here, $\theta_{\text {ref }}=R_{\text {ref }} / z_{0}$ is the angular refraction radius ${ }^{8}$ and $\theta_{\mathrm{s}}$ the source angular radius. In this expression, Narayan (1992) assumed that $z_{0} \ll z_{1}$, therefore $z_{0}+z_{1} \approx z_{1}$. We use the following expression, which is formally identical to the previous one for $z_{0} \ll z_{1}$ but can also be used when $z_{0}$ is not negligible:

$$
\begin{aligned}
m & \approx\left[\frac{R_{\mathrm{diff}}}{R_{\mathrm{F}}}\right]^{1 / 3}\left[\frac{R_{\mathrm{ref}}}{2 \pi R_{\mathrm{S}}}\right]^{7 / 6} \\
& \approx 0.035\left[\frac{\lambda}{1 \mu \mathrm{m}}\right]\left[\frac{z_{0}}{1 \mathrm{kpc}}\right]^{-\frac{1}{6}}\left[\frac{R_{\mathrm{diff}}}{1000 \mathrm{~km}}\right]^{-\frac{5}{6}}\left[\frac{r_{\mathrm{s}} / z_{1}}{R_{\odot} / 10 \mathrm{kpc}}\right]^{-\frac{7}{6}} .
\end{aligned}
$$

This relation can be qualitatively justified by noticing firstly that $R_{\text {diff }}$ quantity has to be considered relatively to $R_{\mathrm{F}}$ when considering its impact on diffraction, and secondly that the convolution of the point-source pattern (see Fig. 7-up) with the projected stellar profile (following expression (17)) makes the contrast decrease when the number of $R_{\text {ref-size domains within }}$ $R_{\mathrm{S}}=r_{\mathrm{S}} z_{0} / z_{1}$ increases. The "exotic" exponents are related to the Kolmogorov turbulence. It should be emphasised that this relation assumes the scintillation is strong and quenched $\left(R_{\mathrm{S}} / R_{\mathrm{F}}>R_{\mathrm{F}} / R_{\text {diff }}>1\right)$.

We checked this relation by performing series of simulations with different phase screens and stellar radii. We generated series of screens with $R_{\text {diff }}=50 \mathrm{~km}$ to $500 \mathrm{~km}$ by steps of $50 \mathrm{~km}$.

8 Narayan uses $\theta_{\text {scatt }}$, which equals $\theta_{\text {ref }} / 2 \pi$. 
For each screen, we considered different sources -at the same geometrical distances- with radii from $0.25 R_{\odot}$ to $1.5 R_{\odot}$ by steps of $0.25 R_{\odot}$ and computed corresponding illumination pattern realisations. The modulation indices were estimated within the fiducial zone for each 2D illumination pattern. In Fig. 8, the thus estimated $m$ for each generated pattern are plotted as a function of the theoretical value expected from expression (23). We note that the modulation has relatively large scatter. This is because the fiducial zone is not large enough, and it contains a limited number of regions with sizes of $R_{\text {ref }}$ (the scale that dominates the light variations). In some cases there are very few distinct dark/luminous regions (as in Fig. 5). The number of such regions within the fiducial domain is $N_{\mathrm{R}} \sim d^{2} / R_{\text {ref }}^{2}$, where $d$ is the size of the fiducial zone. After discarding the cases with $N_{\mathrm{R}}<5$, we computed the mean and root mean square of the effective $m$ values, for each series of configurations with the same theoretical modulation index. Figure 8 shows that relation (23) is satisfied for a modulation index smaller than 0.15 . The higher values of $m$ are systematically underestimated in our simulation owing to the limitation of the screen size, which has a stronger impact on the number of large dark/luminous regions in this part of the figure. This number is indeed smaller (though it is larger than 5), and the chances for sampling the deepest valleys and the highest peaks are fewer, therefore biasing the modulation index towards low values.

Therefore, by measuring the modulation index from an observed light curve and using an estimate of star type (i.e. radius) and distance $z_{0}+z_{1} \approx z_{1}$, we can constrain $R_{\text {diff }}(\lambda)$ from this relation $(23)^{9}$, even with poor knowledge of the screen's distance $z_{0}$, considering the slow dependence with this parameter. This technique allowed us to infer constraints in Habibi et al. (2011) on the gas turbulence within galactic nebulae $\left(z_{0} \sim 80-190 \mathrm{pc}\right.$ and $z_{1}=8 \mathrm{kpc}$ ), and upper limits on hidden turbulent gas within the galactic halo (assuming $z_{0} \sim 10 \mathrm{kpc}$ and $z_{1}+z_{0}=62 \mathrm{kpc}$ ).

\subsection{Information from the light curves}

If a light curve is long enough (or equivalently if the observation time is long enough), its series of light measurements represents an unbiased subsample of the 2D pattern. For instance, the left hand panels of Fig. 9 represent the illumination pattern of a point-like source and three associated light curves. Here, $R_{\text {ref }} \approx 28000 \mathrm{~km}$ and the modulation index $m_{\text {point }}=1.18$. The light curves are extracted from three horizontal parallel lines with lengths of $\sim 3.5 \times 10^{5} \mathrm{~km}$. The corresponding time scale depends on the relative transverse velocity. The lines are selected far from each other in order not to be affected by the fluctuations of the same regions. Modulation indices along the light curves differ from the 2D's by less than $5 \%$. When the modulation is characterised by both $R_{\text {ref }}$ and $R_{\text {diff }}$, a light curve that spans a few $R_{\text {ref }}$ is a sample of the 2D screen, which is large enough to provide a good approximation of the scintillation modulation index for a point-like source. The right hand panels of Fig. 9 show the 2D pattern for an extended source with $R_{\mathrm{S}} \approx 41000 \mathrm{~km}$. Here, $R_{\mathrm{S}}>R_{\text {ref }}$ and the flux fluctuations are smoothed, characterised by the unique length scale $R_{\text {ref }}$, and have a much smaller modulation index $m_{\text {extended }}=0.04$. The light curves are extracted in the same way as the point-like source within the corresponding restricted fiducial zone (see Fig. 5), therefore they span $\sim 2.5 \times 10^{5} \mathrm{~km}$ and statistically include a little bit less than $10 R_{\text {ref-scale variations. Because of this statistically short }}$

\footnotetext{
9 In Habibi et al. (2011), we used a simplified relation, with no significant impact on the resulting constraints.
}
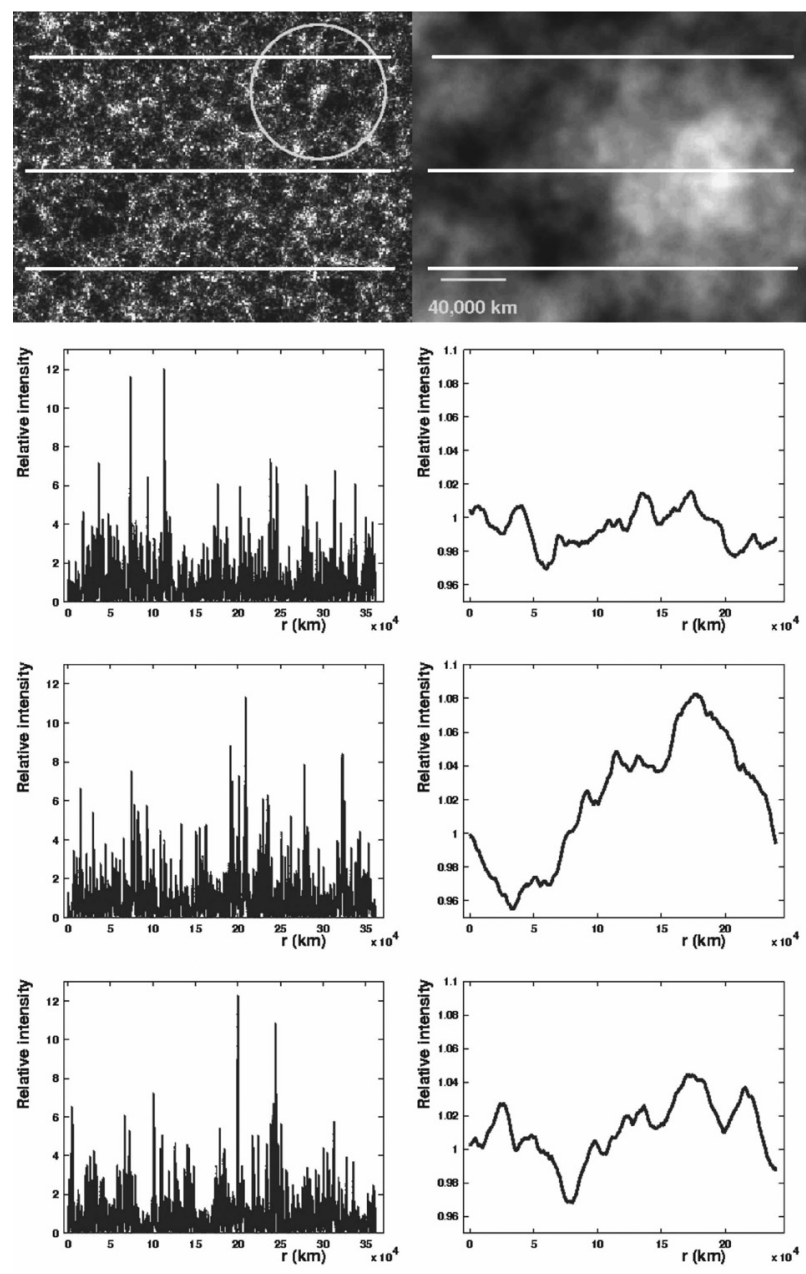

Fig. 9. Light curves extracted along the 3 horizontal white lines for two illumination patterns. Left column: 2D pattern from a point-like source in $K_{\mathrm{s}}$ band with $R_{\text {diff }}=300 \mathrm{~km}$ and $R_{\text {ref }} \approx 28000 \mathrm{~km}$. The modulation indices of the three light curves differ by less than $5 \%$ from the 2D pattern modulation index. Right column: the illumination pattern for an extended source with $R_{\mathrm{s}} \approx 41000 \mathrm{~km}$, through the same refractive screen. The modulation indices fluctuate by more than $30 \%$ around the 2D pattern index, implying the necessity of longer light curves for a better statistical representativity. The distance scale is common to both patterns. The circle shows the projected star disk. The 3 light curves from the right column are not completely decorrelated, because of their common proximity to the same large positive fluctuation.

length, the light-curve-to-light-curve estimates of $m_{\text {extended }}$ typically fluctuate by $\sim 1 / \sqrt{10} \approx 30 \%$. The fluctuations on $m_{\text {extended }}$ estimates can only be reduced with longer light curves. To get a precision value of $5 \%$ on $m_{\text {extended }}$, the light curve should indeed be as long as $\sim 400 \times R_{\text {ref }}$. As an example, if we observe through a turbulent gaseous core with $R_{\text {diff }}=200 \mathrm{~km}$ in B68 nebula located at $z_{0}=80 \mathrm{pc}$ at $\lambda=2.16 \mu \mathrm{m}\left(R_{\text {ref }} \sim 27000 \mathrm{~km}\right)$, and assuming $V_{\mathrm{T}} \sim 20 \mathrm{~km} \mathrm{~s}^{-1}$, an observing time of $\sim 150 \mathrm{~h}$ is needed to measure the modulation index with this precision of $5 \%$. When searching for unseen turbulent media located at unknown distances from us, the diffraction and refraction radii are unknown, and we can only obtain a probability distribution of the observation time for a requested precision on the modulation index.

The other important information carried by the light curves are the characteristic time scale between peaks $t_{\text {ref }}=R_{\text {ref }} / V_{\mathrm{T}}$, associated to the refraction radius (Fig. 7 upper left), a correlation 

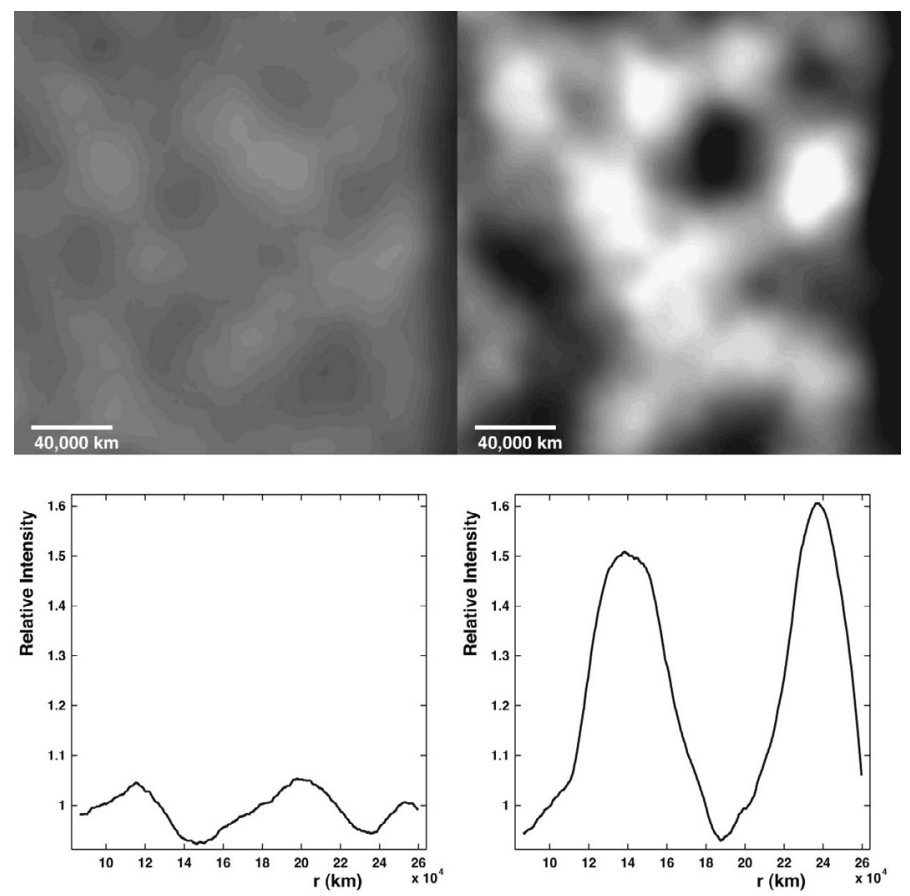

Fig. 10. Simulation of illumination scintillation patterns with associated light curves from an extended source assuming a refractive screen with non-Kolmogorov turbulences (left $\beta=3.1$, right $\beta=3.9$, see text). Here $R_{\text {diff }}=100 \mathrm{~km}, R_{\text {ref }} \approx 8 \times 10^{4} \mathrm{~km}$ and the projected star radius is $R_{\mathrm{S}} \sim 0.36 R_{\text {ref }}=28800 \mathrm{~km}$.

duration $t_{\mathrm{S}}=R_{\mathrm{S}} / V_{\mathrm{T}}$, associated to the source radius (Fig. 7 upper right) and possibly $t_{\text {diff }}=R_{\text {diff }} / V_{\mathrm{T}}$, associated to the small diffractive speckle structure (Fig. 7 lower left); the latter could be detected in exceptionally favourable cases (source with very small angular size, assuming the strong diffractive scintillation regime) with a powerful detection setup such as $\operatorname{LSST}^{10}$. The time power spectrum of the expected stochastic light curve should show a slope break around frequency $t_{\text {ref }}^{-1}$, and possibly around $t_{\text {diff }}^{-1}$ (Goodman \& Narayan 1985), which should allow one to distinguish it from purely randomly fluctuating light curves due to photometric noise, and to extract constraints on the scintillation configuration. Estimating $t_{\mathrm{s}}$ would be challenging, since the extended source acts as a low passband filter on the point-source pattern; therefore, the imprint of the projected radius is essentially within the attenuation of the light curve autocorrelation (Goodman \& Narayan 2006), and more specifically - as mentioned above - within the modulation index, that can be polluted by many observational artefacts. These potentialities of the time studies need more investigation and should be discussed in more detail in a forthcoming paper.

\section{Probing various turbulence laws}

Up to now, we have focussed on the standard Kolmogorov turbulence. In this section, we investigate a possible deviation from

\footnotetext{
10 The detection condition would be $r_{\mathrm{s}} / z_{1} \lesssim 10 \times R_{\mathrm{diff}} / z_{0}$ (for which the projected stellar disk includes less than $\sim 100$ speckle spots), assuming a setup able to sample the target star with $\lesssim 1 \%$ photometric precision every few seconds. The scintillation of an $A 0$ type star $\left(R=2.4 R_{\odot}\right)$ in the LMC (magnitude $V=19.4$ ) seen through a screen with $R_{\text {diff }}=100 \mathrm{~km}$ at distance $30 \mathrm{pc}$ is an example of such a favourable configuration, which could be discovered by the LSST (Abell 2009).
}
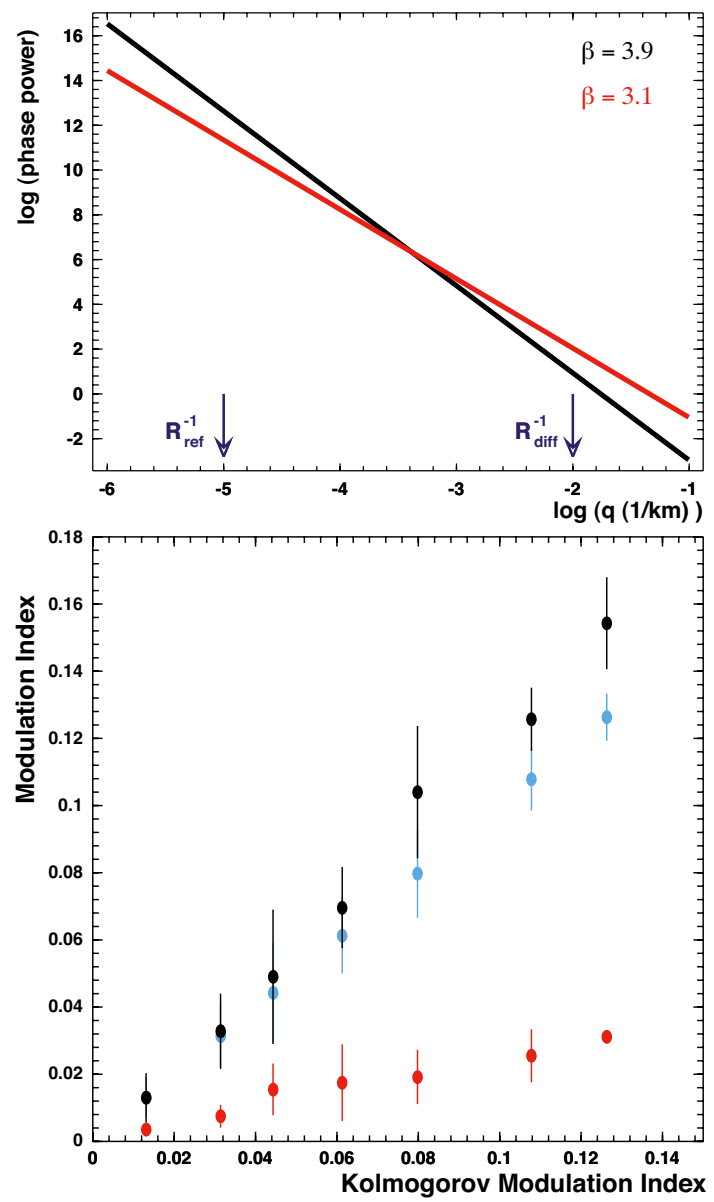

Fig. 11. Top: two different phase screen power laws. Bottom: the corresponding modulation indices as a function of the expected modulation index for the Kolmogorov turbulence. The 3 indices plotted at a given abscissa correspond to screens with $\beta=3.1$ (red), 3.67 (Kolmogorov, blue, along the diagonal), and 3.9 (black) with the same $R_{\text {diff }}$. As the power law gets steeper, a larger modulation is expected. Blue dots show the Kolmogorov turbulence case.

the Kolmogorov turbulence law ${ }^{11}$. We chose two different phase spectra with $\beta=3.1$ and $\beta=3.9$ in relation (4). To study the corresponding scintillation modes, we generated two series of phase screens according to the spectra and computed the illumination patterns from an extended source with $R_{\mathrm{S}} \sim 0.36 R_{\text {ref }}$ through each of them. The patterns are represented in Fig. 10 with corresponding light curve samples. For both images, $R_{\text {diff }}=100 \mathrm{~km}$, $R_{\mathrm{F}}=1150 \mathrm{~km}$, and $R_{\text {ref }} \approx 8 \times 10^{4} \mathrm{~km}$. The pattern with $\beta=3.1$ (left) shows a small modulation index $m(3.1)=0.04$, while the pattern with $\beta=3.9$ (right) shows a much larger modulation in$\operatorname{dex} m(3.9)=0.22$. As can be seen from its visual aspect, the turbulence with a higher exponent produces stronger contrast on large scales compared to the other one $(\beta=3.1)$. To understand the origin of the difference, we compared the two phase spectra at the top of Fig. 11. The steeper spectrum $(\beta=3.9)$ has more power for fluctuations on large scales. Moreover, by integrating Eq. (4), we computed that the total power distributed from $R_{\text {ref }}$ to $R_{\text {diff }}$ is about an order of magnitude larger for $\beta=3.9$ than for $\beta=3.1$. Therefore, the larger the $\beta$ exponent is the stronger flux fluctuations are produced. As a conclusion, the detection of scintillation should be easier for turbulences with steeper spectrum,

${ }^{11}$ For the observed supersonic turbulence (with $\beta>11 / 3$ ) see Larson (1981). 
because a larger modulation index is expected. This is illustrated at the bottom of Fig. 11 where we plot the modulation indices produced by three screens with different $\beta$ values (including the Kolmogorov case), as a function of the "classical" Kolmogorov turbulence expected index. In this plot, each abscissa corresponds to a distinct $R_{\text {diff }}$ value, which is common to the three screens. By increasing $\beta$, we increase the modulation, especially for high $m$ values.

\section{Discussion: guidelines provided by the simulation}

The observations analysed in (Habibi et al. 2011) were intrepreted by using our simulation pipeline. But we have used our simulation not only to establish connections between the observed light curves and the scintillation configuration, but also to define observing strategies as follows.

Firstly, a correct sensitivity to the scintillation needs the ability to sample, with $<1 \%$ photometric precision at a sub-minute rate, the light curves of small distant background stars $(M \sim$ 20-21), which have a projected radius small enough to allow for a modulation index of a few percent (typically $<R_{\odot}$ at $10 \mathrm{kpc}$ ). Our study of the time coherency shows that the usual large passband filters can be used without significant loss of modulation index. Since the optical depth of the process is unknown, a large field of view seems necessary for the exploratory observations, either toward extragalactic stellar sources within LMC or SMC or through known gaseous nebulae. To summarise, an ideal setup for searching for scintillation with series of sub-minute exposures would be a $\sim 4 \mathrm{~m}$ class telescope equipped with a fast readout wide field camera and a standard filter (optical passband to search for invisible gas towards extragalactic sources, infrared to observe stars through visible dusty nebulae).

Secondly, our work on the simulation provides us with a guideline to find an undisputable signature of scintillation. The first possibility consists in the search for chromatic effects. Subtle chromatic effects betwen the different regimes (associated to different time scales) have been shown, but will probably be hard to observe. Figure 7 (left and centre) shows the expected speckle image from a point source. The position/size of the small speckles (characterised by $R_{\text {diff }}(\lambda)$ ) are very sensitive to the wavelength, and it is clear that a desynchronisation of the maxima would be expected when observing such an idealised point source through different (narrow) passbands. But that real sources have a much larger projected radius than the speckle size completely screens this chromatic effect. The impact of the wavelength on the position/size of the wide (refractive) spots (Fig. 7-right) is much weaker (following $\lambda^{-1 / 5}$ according to the combination of expressions (3) and (9)). Therefore, only a weak chromatic effect is expected from an extended source, even when observing with two very different passbands. For a clear signature of scintillation, it seems easier to take advantage of the rapidly varying luminosity with the observer's position within the illumination pattern. As a consequence, simultaneous observations with two $\sim 4 \mathrm{~m}$ class telescopes at a large separation (few $10^{3} \mathrm{~km}$ ) would sample different regions of the illumination patterns (see Fig. 7 bottom right), and therefore measure (at least partially) decorrelated light curves as shown in Fig. 9 (right). The decorrelation will be complete if the distance between the two telescopes is greater than $2 \times \max \left(R_{\text {ref }}, R_{\mathrm{S}}\right)$. A single observation of such a decorrelation will be sufficient to definitely confirm the discovery of a propagation effect that cannot be mimicked by an intrinsic variability.

\section{Conclusions and perspectives}

Through this work, we have simulated the phase delay induced by a turbulent refractive medium on the propagation of a wave front. We discussed the computational limitations of sampling the phase spectrum and to obtaining large enough illumination patterns. These limitations will be overriden in the near future with increasing computing capabilities. The illumination pattern on the observer's plane has been computed for the promising strong regime of scintillation, and the effects of the source spatial and time coherencies have been included. We have established the connection between the modulation index (as an observable) of the illumination pattern with the geometrical parameters of the source and the strength of the turbulence (quantified by $R_{\text {diff }}$ ). Furthermore, we showed that when the spectral index of the turbulence increases (as in the case of supersonic turbulence), the detection of scintillating light curves should be easier.

These simulation studies and, more specifically, the modulation index topic were successfully used in our companion paper (Habibi et al. 2011) to interpret our light curve test observations of stars located behind known galactic nebulae and of stars from the Small Magellanic Cloud, in the search for hypothetical cold molecular halo clouds.

Time scales, such as $t_{\text {ref }}=R_{\text {ref }} / V_{\mathrm{T}}$ and $t_{\mathrm{S}}=R_{\mathrm{S}} / V_{\mathrm{T}}$ (where $V_{\mathrm{T}}$ is the relative velocity between the cloud and the line of sight), are observables that we plan to study further and in detail. Their extraction can be done through analysing the time power spectrum of the light curves, and it should give valuable information on the geometrical configuration, as well as on the turbulent medium.

The observing strategy has been refined through the use of the simulation, and we showed that observing desynchronised light curves simultaneously measured by two distant four-metre class telescopes would provide an unambiguous signature of scintillation as a propagation effect.

Acknowledgements. We thank J.-F. Lestrade, J.-F. Glicenstein, F. Cavalier, and P. Hello for their participation in preliminary discussions. We wish to thank our referee, Prof. B. J. Rickett, for his very careful review, which helped us to significantly improve the manuscript.

\section{References}

Abell P. A., et al. (LSST Science Collaboration) 2009, LSST Science Book, submitted [arXiv:0912.0201]

Born, M., \& Wolf, E. 2002, Principles of Optics (Cambridge: University Press), 7 th edn.

Coles, Wm. A., Filice, J. P., Frehlich R. G., \& Yadlowsky, M. 1995, Appl. Opt., 34, 2089

Goodman, J., \& Narayan, R. 1985, MNRAS, 214, 519

Goodman, J., \& Narayan, R. 2006, ApJ, 636, 510

Gwinn, C. R., Britton, M. C., Reynolds, J. E. et al. 1998, ApJ, 505, 928

Habibi, F. 2011, Ph.D. Thesis, Paris-Sud and Sharif Universities

Habibi, F., Ansari, R., Moniez, M., \& Rahvar, S. 2011, A\&A, 525, A108

Hamidouche, M., \& Lestrade, J.-F. 2007, A\&A, 468, 193

Larson, R. B. 1981, MNRAS, 194, 809

Lovelace, R. V. E. 1970, Ph.D. Thesis, Cornell University

Lyne, A. G., \& Graham-Smith, F. 1998, Pulsar Astronomy (Cambridge University Press)

McGaugh, S. S., Schombert, De Block, W. J. G., \& Zagursky, M. J. 2010, ApJ, 708, L14

Moniez, M. 2003, A\&A, 412, 105

Narayan, R. 1992, Phil. Trans. Roy. Soc. Lond. A, 341, 151

Pfenniger, D., \& Combes, F. 1994, A\&A, 285, 94

Pfenniger, D., \& Revaz, Y. 2005, A\&A, 431, 511

Rickett, B. J. 1986, ApJ, 307, 564

Rumsey, V. H. 1975, Radio Sci., 10, 107

Sieber, W. 1982, A\&A, 113, 311

Tatarskii, V. I., \& Zavorotnyi, V. U. 1980, Progr. Opt., 18, 207

Uscinski, B. J. 1977, The elements of wave propagation in random media (New York: McGraw-Hill) 